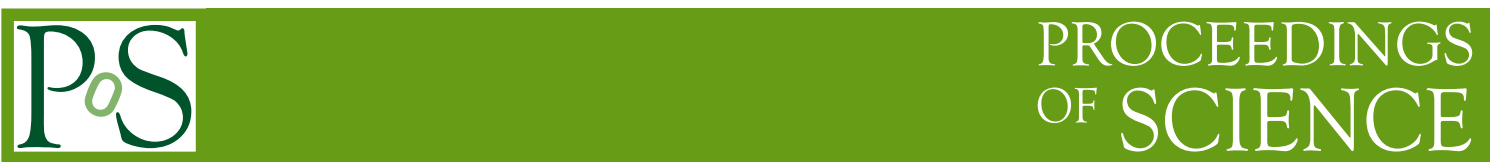

\title{
Hadron gas with repulsive mean field
}

\author{
Pasi Huovinen* \\ Institute of Theoretical Physics, University of Wroclaw, 50204 Wroctaw, Poland \\ E-mail: pasi.huovineneift.uni.wroc.pl
}

\section{Peter Petreczky}

Physics Department, Brookhaven National Laboratory, Upton, NY 11973, USA

E-mail: petreczk@quark.phy.bnl.gov

We study the QCD equation of state, and fluctuations of baryon number and strangeness using the hadron resonance gas model with repulsive mean field. We find that including both the predicted but not observed resonances, a.k.a. missing states, and the repulsive mean field into the resonance gas model leads to better description of the lattice results. The repulsive mean field is particularly important for the higher order baryon number fluctuations.

XIII Quark Confinement and the Hadron Spectrum - Confinement2018

31 July - 6 August 2018

Maynooth University, Ireland

${ }^{*}$ Speaker. 


\section{Introduction}

Below the chiral crossover at zero net baryon density QCD thermodynamics can be well approximated by the hadron resonance gas (HRG) model. This model is based on the idea that the effect of interactions in hadron gas can be mimicked by including resonances as additional free particles in hadron gas - an idea, which can be justified using the relativistic virial expansion based on the S-matrix approach [1]. In most interactions, after summation over spin and isospin channels, the second virial coefficient is dominated by the resonance contribution, and thus the interacting gas of hadrons can be approximated as a gas of non-interacting hadrons and hadronic resonances [2]. The validity of the HRG model is further corroborated by the equation of state obtained in lattice QCD calculations, which agrees well with the HRG equation of state (see e.g. Refs. [3, 4, 5, 6]). The fluctuations and correlations of conserved charges, defined as derivatives of pressure with respect to chemical potentials, have also been studied in the HRG model (see e.g. Ref. [7]).

However, the applicability of HRG must have its limits. The cancellation of non-resonant contributions to thermodynamics is somewhat accidental [8] and does not happen for all thermodynamic quantities [9]. There are also hadronic interactions which are not dominated by resonances, e.g. there are no resonances in nucleon-nucleon scatterings. Last but not least, the HRG model is based on free particle properties, but close to the chiral transition there are in-medium modifications to hadron properties $[10,11]$. Therefore, it is important to check the HRG model by comparing it to recent lattice QCD calculations of different thermodynamic quantities.

It is known that for higher order $(>2)$ fluctuations the agreement between lattice and HRG is not good for temperatures close to the chiral transition. It has been argued that this is not a signal of approaching transition, but rather due to repulsive baryon-baryon interactions which the HRG model does not describe $[12,13,14]$. If that is the case, the repulsive baryon-baryon interactions must be included in the model when HRG is extended to non-zero baryon densities, since the larger the baryon density, the larger the effect of repulsive interactions. As a step towards finding a convenient description of baryon repulsion, we study the trace anomaly and fluctuations of baryon number and strangeness using HRG with repulsive mean field.

\section{Nucleon gas with repulsive mean field and virial expansion}

According to the virial expansion, pressure of the interacting nucleon gas can be written as [15]

$$
p(T, \mu)=p_{0}(T) \cosh (\beta \mu)+2 b_{2}(T) T \cosh (2 \beta \mu), \beta=1 / T,
$$

where

$$
p_{0}(T)=\frac{4 M^{2} T^{2}}{\pi^{2}} K_{2}(\beta M)
$$

is the pressure of free nucleon gas at zero chemical potential, and $b_{2}(T)$ is the second virial coefficient. The second virial coefficient can be written as

$$
b_{2}(T)=\frac{2 T}{\pi^{3}} \int_{0}^{\infty} d E\left(\frac{M E}{2}+M^{2}\right) K_{2}\left(2 \beta \sqrt{\frac{M E}{2}+M^{2}}\right) \frac{1}{4 i} \operatorname{Tr}\left[S^{\dagger} \frac{d S}{d E}-\frac{d S^{\dagger}}{d E} S\right],
$$

where $S$ is the scattering S-matrix and $E$ the kinetic energy in the lab frame. Furthermore, $M$ is the nucleon mass and $K_{2}(x)$ is the Bessel function of second kind. The virial coefficient $b_{2}(T)$ can be 
evaluated using the experimentally measured phase shifts to parametrise the $S$-matrix, and it turns out that $b_{2}(T)$ is negative [15], see Fig. 1.

For the comparison with the mean-field approach it is convenient to write the pressure as

$$
p(T, \mu)=p_{0}(T)\left(\cosh (\beta \mu)+\bar{b}_{2}(T) K_{2}(\beta M) \cosh (2 \beta \mu)\right),
$$

where

$$
\bar{b}_{2}(T)=\frac{2 T b_{2}(T)}{p_{0}(T) K_{2}(\beta M)}
$$

is the reduced virial coefficient.

In the mean-field approach pressure can be written in Boltzmann approximation as [15]

$$
p(T, \mu)=T\left(n_{b}+\bar{n}_{b}\right)+\frac{K}{2}\left(n_{b}^{2}+\bar{n}_{b}^{2}\right),
$$

where $n_{b}$ and $\bar{n}_{b}$ are the densities of nucleons and anti-nucleons, respectively, defined by the following self-consistent relations:

$$
n_{b}=4 \int \frac{d^{3} p}{(2 \pi)^{3}} e^{-\beta\left(E_{p}-\mu+U\right)}, \bar{n}_{b}=4 \int \frac{d^{3} p}{(2 \pi)^{3}} e^{-\beta\left(E_{p}+\mu+\bar{U}\right)}, E_{p}^{2}=p^{2}+M^{2} .
$$

Here $U=K n_{b}$ and $\bar{U}=K \bar{n}_{b}$ are the mean-field potentials for nucleons and anti-nucleons. The chemical potential corresponding to the net nucleon density is denoted by $\mu$. This form of the pressure ensures thermodynamic consistency, i.e. $\partial p / \partial \mu=n_{b}-\bar{n}_{b}$. Since we are mostly interested in the region of not too high baryon densities we expand the above expressions in $\beta U=\beta K n_{b}$ and $\bar{U}=K \bar{n}_{b}$, and keep only the leading order terms in $K$. This simplifies the pressure to

$$
p(T, \mu)=T\left(n_{b}^{0}+\bar{n}_{b}^{0}\right)-\frac{K}{2}\left(\left(n_{b}^{0}\right)^{2}+\left(\bar{n}_{b}^{0}\right)^{2}\right),
$$

where $n_{b}^{0}\left(\bar{n}_{b}^{0}\right)$ is the free nucleon (anti-nucleon) density. Equation (2.8) can also be written as

$$
p(T, \mu)=\frac{4 T^{2} M^{2}}{\pi^{2}} K_{2}(\beta M) \cosh (\beta \mu)-4 K \frac{T^{2} M^{4}}{\pi^{4}} K_{2}^{2}(\beta M) \cosh (2 \beta \mu),
$$

which is very similar to the virial expansion pressure Eq. (2.4). By comparing these two results, one can determine the value of the mean field coefficient $K$ in a limited temperature range. Since $\bar{b}_{2}(T)$ turns out to be negative, $K>0$, and the mean field is repulsive. The temperature dependence of $\bar{b}_{2}$ is shown in Fig. 1 and it turns out to be relatively mild except maybe at the highest temperature. The value of $\bar{b}_{2}$ is consistent with $K=250 \mathrm{MeV} / \mathrm{fm}^{3}$. The largest value allowed for $K$ by the virial expansion is around $K=450 \mathrm{MeV} / \mathrm{fm}^{3}$.

It is straightforward to generalise the mean-field approach to a multicomponent system if one assumes that the repulsive mean-field is the same for all ground state baryons [15], and the baryon resonances are not affected by the mean field. Within this approach we calculated baryon number fluctuations defined as derivatives of the pressure with respect to baryon chemical potential [15]

$$
\chi_{n}^{B}=T^{n} \frac{\partial^{n} p / T^{4}}{\partial \mu_{B}^{n}} .
$$




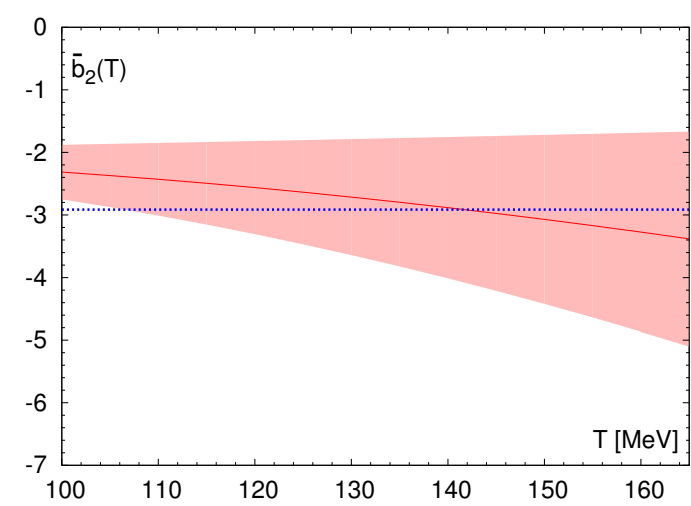

Figure 1: The reduced second virial coefficient $\bar{b}_{2}$ as function of temperature (red), and the corresponding coefficient for mean field parameter $K=250 \mathrm{MeV} / \mathrm{fm}^{3}$ (blue).

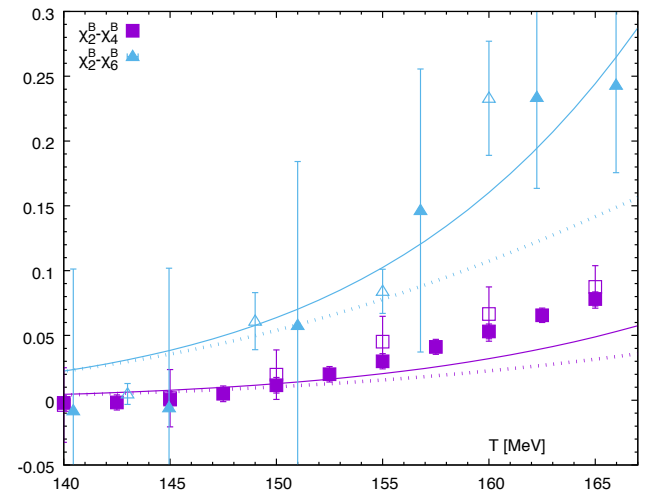

Figure 2: The difference between fourth and second order baryon number fluctuations (squares) and the the sixth and second order baryon number fluctuations (triangles). The dashed lines correspond to the exact mean-field calculations, while the solid line to the expanded mean field (see text). The filled symbols correspond to lattice results of Ref. [16]. The open symbols are the lattice data for $\chi_{4}^{B}$ from Ref. [17] and $\chi_{6}^{B}$ from Ref. [18], respectively.

In Fig. 2 we show the differences of the baryon number fluctuations, $\chi_{4}^{B}-\chi_{2}^{B}$ and $\chi_{6}^{B}-\chi_{2}^{B}$ obtained in HRG model with the mean field and the value of $K=450 \mathrm{MeV} / \mathrm{fm}^{3}$. As seen, the model can qualitatively explain these differences, but at quantitative level it underpredicts the lattice data even if the value of $K$ is the largest the experimental data allows. We note that at temperatures above $150 \mathrm{MeV}$ the expanded mean-field expression (2.8) is no longer accurate and one should use the exact mean-field expressions with particle densities determined self-consistently, Eq. (2.7) [15]. The exact mean-field results are shown as dashed lines in the figure.

\section{Comparison with lattice QCD}

After fixing the value of the mean field parameter, we compare the HRG calculations with repulsive mean field with lattice QCD results on the trace anomaly, fluctuations of baryon number up to sixth order, and second order strangeness fluctuations. First we note that there are many baryon resonances which are not experimentally observed but predicted by quark models and lattice QCD, so-called missing states. It was found that inclusion of these states in the HRG description improves the description of the strangeness fluctuations and baryon-strangeness correlation [19]. Therefore, in our analysis we will include the missing baryons from the quark model calculations of Refs. [20, 21], missing mesons from Ref. [22], and label the corresponding results as QM-HRG, whereas the HRG based on the Particle Data Group's (PDG) resonance list [23] is labeled PDGHRG. The difference in the mass spectrum of these two approaches is depicted in Fig. 3.

In our previous analysis we assumed that the baryon resonances are not affected by the mean field [15]. This is not realistic because if the density of ground state baryons is reduced there will be 
also fewer baryon resonances in the system. Therefore, in the present analysis we include the effect of the repulsive mean field on baryon resonances as well, but assume that baryon resonances do no contribute to the strength of the mean field ${ }^{1}$. We also use a more realistic value for the mean-field parameter, $K=250 \mathrm{MeV} / \mathrm{fm}^{3}$, and show the results for an exact mean-field calculation only.

In Fig. 4 we show our calculations for the trace anomaly, $\varepsilon-3 P$, compared with the lattice results $[4,5]$ at zero baryon chemical potential, $\mu_{B}=0$. As expected the missing states have a clear effect on the trace anomaly improving the fit to the lattice data: These states lead to significant increase of the trace anomaly around temperatures of $150 \mathrm{MeV}$ and higher compared to the HRG calculations with only PDG states (PDG-HRG). The HRG calculations with repulsive mean field are shown as dashed lines. The effect of the repulsive interactions turns out to be relatively small, because the contribution of baryons to the equation of state is small too. The situation will be different at sufficiently large $\mu_{B}$, when the contributions of mesons and baryons to the equation of state are comparable.

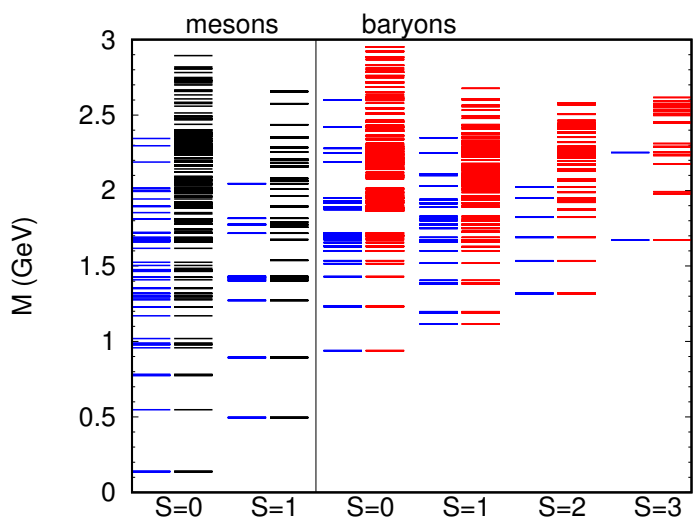

Figure 3: The mass spectrum of non-strange and strange mesons, non-strange baryons, and baryons of strangeness $S=1,2$ and 3 as given in Particle Data Group's 2016 summary tables (blue) [23], or by PDG but augmented by additional states from quark model calculations from Ref. [22] (mesons,black) and Refs. [20, 21] (baryons, red)

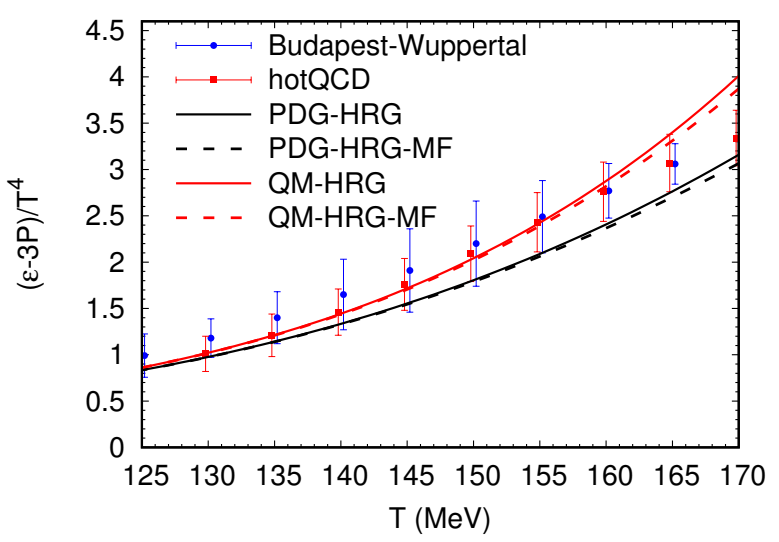

Figure 4: The trace anomaly in HRG model with the Particle Data Group (PDG-HRG) and Quark Model (QM-HRG) lists of resonances compared to the lattice results $[4,5]$. The dashed lines correspond to $\mathrm{HRG}$ model with repulsive mean field.

In the left panel of Fig. 5 we show our results for second order baryon number fluctuations, again for QM-HRG and PDG-HRG with and without the effect of the repulsive mean field. The effect of the missing states is clearly visible and improves the fit to lattice data around 140-150 $\mathrm{MeV}$ temperature, but overshoots the lattice results at higher temperatures. The repulsive mean field has an opposite effect and is comparable in size. Therefore, it restores the agreement with the lattice data. The second order strangeness fluctuations are shown in the right panel of Fig. 5. The need for more resonance states to fit the data is now even clearer than in the case of baryon number fluctuations, and even the full quark model spectrum of states hardly reaches the lattice data. The mean field, on the other hand, affects the strangeness fluctuations less, since they are dominated by kaons and other strange mesons which are not affected by baryonic interactions.

\footnotetext{
${ }^{1}$ We count members of baryon octet and decuplet as ground state baryons, and all other baryon states as resonances.
} 

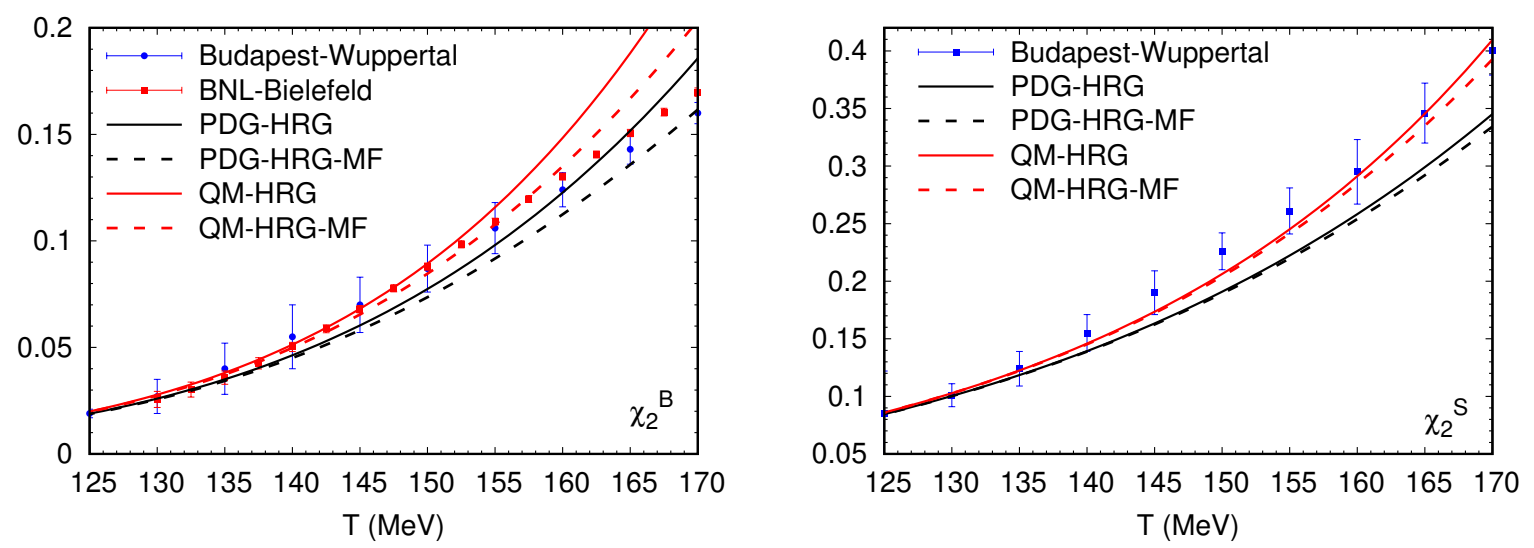

Figure 5: The second order baryon number (left) and strangeness (right) fluctuations in HRG model with the Particle Data Group (PDG-HRG) and Quark Model (QM-HRG) lists of resonances compared to the lattice results $[17,16]$. The dashed lines correspond to HRG model with repulsive mean field.
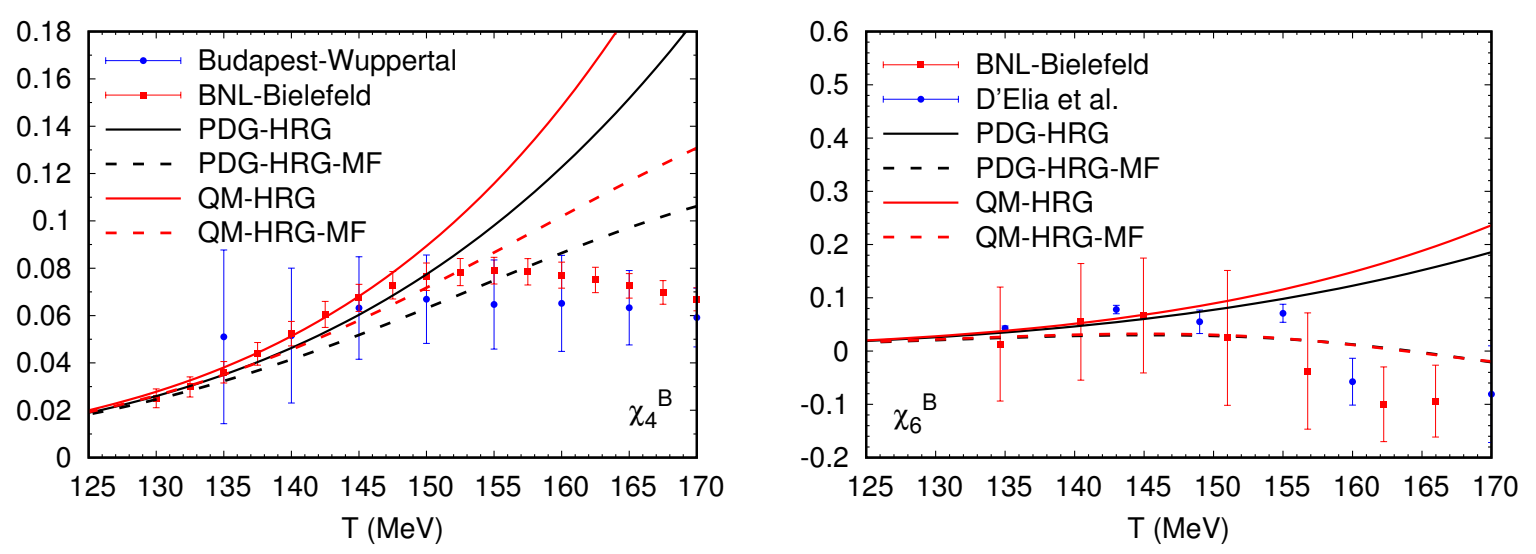

Figure 6: The fourth order (left) and sixth order (right) baryon number fluctuations in HRG-QM and HRGPDG models. The lattice results for $\chi_{4}^{B}$ are from Refs. [17, 16], while the lattice results for $\chi_{6}^{B}$ are from $[18,16]$. The dashed lines correspond to HRG model with repulsive mean field.

Higher order baryon number fluctuations are more sensitive to the effects of the repulsive interactions than the second order fluctuations as shown in Fig. 6. The QM-HRG model again overpredicts the lattice results and lies significantly above the PDG-HRG result. The repulsive interactions reduce the HRG prediction and lead to reasonable agreement with the lattice data. Note that the effects of the repulsive mean field are now larger than in the previous analysis shown in Fig. 2 even though we use smaller value of $K$. This is because the resonances are also affected by the repulsive mean field. Note also that when the effect of the mean field is included, the sixth order baryon number fluctuation is almost independent of the mass spectrum of resonances.

\section{Conclusion}

We studied the equation of state and fluctuations of baryon number and strangeness within the HRG framework, where the effect of the repulsive baryon-baryon interactions are included using 
the mean-field approach. We also studied the effects of missing resonances on these quantities. We have found that the missing states lead to significant increase of thermodynamic quantities. In the case of baryon number fluctuations missing states cause the HRG model to overshoot the lattice results for $T \geq 150 \mathrm{MeV}$. The repulsive mean field has the opposite effect. The effects of repulsive interactions are small for the trace anomaly and strangeness fluctuations, but are significant for baryon number fluctuations, where they are needed to bring the HRG calculations in agreement with the lattice results. This implies that when extending the HRG model to calculate the equation of state at non-zero baryon density the repulsive interactions have to be taken into account.

\section{Acknowledgments}

This work was supported by National Science Center, Poland, under grant Polonez DEC2015/19/P/ST2/03333 receiving funding from the European Union's Horizon 2020 research and innovation program under the Marie Skłodowska-Curie grant agreement No 665778, and by U.S.Department of Energy under Contract No. DE-SC0012704.

\section{References}

[1] R. Dashen, S. K. Ma and H. J. Bernstein, S Matrix formulation of statistical mechanics, Phys. Rev. 187 (1969) 345.

[2] R. Venugopalan and M. Prakash, Thermal properties of interacting hadrons, Nucl. Phys. A 546 (1992) 718 .

[3] S. Borsanyi et al. [Wuppertal-Budapest Collaboration], Is there still any $T_{c}$ mystery in lattice QCD? Results with physical masses in the continuum limit III, JHEP 1009 (2010) 073 [arXiv:1005.3508 [hep-lat]].

[4] S. Borsanyi, Z. Fodor, C. Hoelbling, S. D. Katz, S. Krieg and K. K. Szabo, Full result for the QCD equation of state with 2+1 flavors, Phys. Lett. B 730 (2014) 99 [arXiv: 1309.5258 [hep-lat]].

[5] A. Bazavov et al. [HotQCD Collaboration], Equation of state in ( 2+1)-flavor QCD, Phys. Rev. D 90 (2014) 094503 [arXiv:1407.6387 [hep-lat]].

[6] A. Bazavov, P. Petreczky and J. H. Weber, Equation of State in 2+1 Flavor QCD at High Temperatures, Phys. Rev. D 97 (2018) 014510 [arXiv:1710.05024 [hep-lat]].

[7] P. Huovinen and P. Petreczky, QCD Equation of State and Hadron Resonance Gas, Nucl. Phys. A 837 (2010) 26 [arXiv:0912.2541 [hep-ph]].

[8] C. Fernández-Ramírez, P. M. Lo and P. Petreczky, Thermodynamics of the strange baryon system from a coupled-channels analysis and missing states, Phys. Rev. C 98 (2018) 044910 [arXiv:1806.02177 [hep-ph]].

[9] P. M. Lo, B. Friman, K. Redlich and C. Sasaki, S-matrix analysis of the baryon electric charge correlation, Phys. Lett. B 778 (2018) 454 [arXiv:1710.02711 [hep-ph] ].

[10] A. Kelly, A. Rothkopf and J. I. Skullerud, Bayesian study of relativistic open and hidden charm in anisotropic lattice QCD, Phys. Rev. D 97 (2018) 114509 [arXiv:1802.00667 [hep-lat]]. 
[11] G. Aarts, C. Allton, D. De Boni, S. Hands, B. Jäger, C. Praki and J. I. Skullerud, Light baryons below and above the deconfinement transition: medium effects and parity doubling, JHEP 1706 (2017) 034 [arXiv:1703.09246 [hep-lat]].

[12] M. Albright, J. Kapusta and C. Young, Baryon Number Fluctuations from a Crossover Equation of State Compared to Heavy-Ion Collision Measurements in the Beam Energy Range $\sqrt{s_{N N}}=7.7$ to 200 GeV, Phys. Rev. C 92 (2015) 044904 [arXiv: 1506.03408 [nucl-th]].

[13] V. Vovchenko, M. I. Gorenstein and H. Stoecker, van der Waals Interactions in Hadron Resonance Gas: From Nuclear Matter to Lattice QCD, Phys. Rev. Lett. 118 (2017) 182301 [arXiv:1609.03975 [hep-ph]].

[14] V. Vovchenko, A. Motornenko, P. Alba, M. I. Gorenstein, L. M. Satarov and H. Stoecker, Multicomponent van der Waals equation of state: Applications in nuclear and hadronic physics, Phys. Rev. C 96 (2017) 045202 [arXiv: 1707.09215 [nucl-th]].

[15] P. Huovinen and P. Petreczky, Hadron Resonance Gas with Repulsive Interactions and Fluctuations of Conserved Charges, Phys. Lett. B 777 (2018) 125 [arXiv:1708.00879 [hep-ph] ].

[16] A. Bazavov et al., The QCD Equation of State to $\mathscr{O}\left(\mu_{B}^{6}\right)$ from Lattice QCD, Phys. Rev. D 95 (2017) 054504 [arXiv:1701.04325 [hep-lat]].

[17] S. Borsanyi, Z. Fodor, S. D. Katz, S. Krieg, C. Ratti and K. K. Szabo, Freeze-out parameters from electric charge and baryon number fluctuations: is there consistency?, Phys. Rev. Lett. 113 (2014) 052301 [arXiv:1403.4576 [hep-lat]].

[18] M. D'Elia, G. Gagliardi and F. Sanfilippo, Higher order quark number fluctuations via imaginary chemical potentials in $N_{f}=2+1$ QCD, Phys. Rev. D 95 (2017) 094503 [arXiv: 1611.08285 [hep-lat] ].

[19] A. Bazavov et al., Additional Strange Hadrons from QCD Thermodynamics and Strangeness Freezeout in Heavy Ion Collisions, Phys. Rev. Lett. 113 (2014) 072001 [arXiv: 1404.6511 [hep-lat] ].

[20] U. Loring, B. C. Metsch and H. R. Petry, The Light baryon spectrum in a relativistic quark model with instanton induced quark forces: The Nonstrange baryon spectrum and ground states, Eur. Phys. J. A 10 (2001) 395 [hep-ph/ 0103289 ].

[21] U. Loring, B. C. Metsch and H. R. Petry, The Light baryon spectrum in a relativistic quark model with instanton induced quark forces: The Strange baryon spectrum, Eur. Phys. J. A 10 (2001) 447 [hep-ph/0103290].

[22] D. Ebert, R. N. Faustov and V. O. Galkin, Mass spectra and Regge trajectories of light mesons in the relativistic quark model, Phys. Rev. D 79 (2009) 114029 [arXiv:0903.5183 [hep-ph]].

[23] C. Patrignani et al. [Particle Data Group], Review of Particle Physics, Chin. Phys. C 40 (2016) 100001 . 